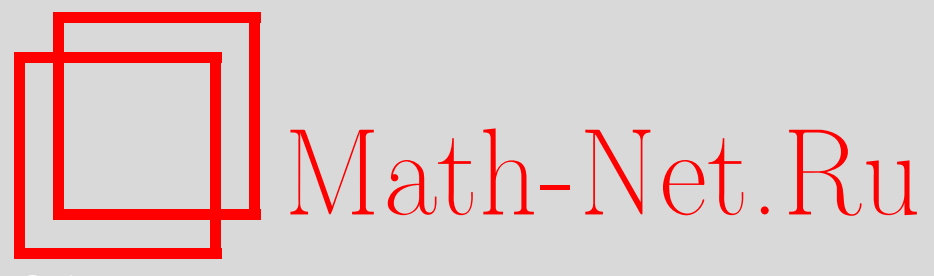

О. И. Мохов, О коммутативных подалгебрах алгебр Вейля, связанных с коммутирующими операторами произвольного ранга и рода, Матем. заметки, 2013, том 94, выпуск 2, 314-316

DOI: https://doi.org/10.4213/mzm9339

Использование Общероссийского математического портала Math-Net.Ru подразумевает, что вы прочитали и согласны с пользовательским соглашением http://www. mathnet.ru/rus/agreement

Параметры загрузки:

IP : 54.174 .149 .18

26 апреля 2023 г., 14:19:16

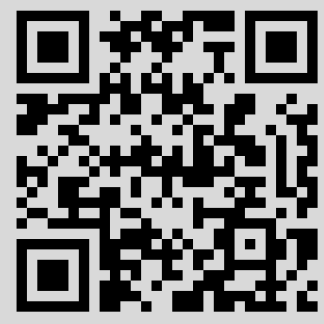




\section{О коммутативных подалгебрах алгебр Вейля, связанных с коммутирующими операторами произвольного ранга и рода}

\section{О. И. Мохов}

В данной заметке построены примеры коммутирующих обыкновенных скалярных дифференциальных операторов с полиномиальными коэффициентами, отвечающих спектральной кривой произвольного рода $g$ и произвольному четному рангу $r=2 k$, а также произвольному рангу вида $r=3 k$ векторного расслоения общих собственных функций коммутирующих операторов над спектральной кривой. На настоящий момент мы не знаем никаких явных примеров коммутирующих обыкновенных скалярных дифференциальных операторов, отвечающих спектральной кривой рода $g>1$ для ранга вида $r=6 s \pm 1, s \geqslant 1$. Для всех остальных значений рода $g$ и ранга $r$ построены явные примеры коммутирующих операторов даже с полиномиальными коэффициентами. Наша гипотеза состоит в том, что существуют коммутирующие обыкновенные скалярные дифференциальные операторы с полиномиальными коэффициентами, отвечающие спектральной кривой произвольного рода $g>1$ и для произвольного ранга вида $r=6 s \pm 1, s \geqslant 1$, но такие примеры пока не построены, - это очень интересная проблема. Хорошо известно, что коммутирующие обыкновенные скалярные дифференциальные операторы с полиномиальными коэффициентами задают коммутативные подалгебры алгебры Вейля $W_{1}$, т.е. алгебры с двумя образующими $p$ и $q$ и соотношением $[p, q]=1$. По лемме Бурхналла-Чаунди [1] любая пара коммутирующих обыкновенных скалярных дифференциальных операторов $L$ и $M$ связана некоторым полиномиальным соотношением $Q(L, M)=0$, определяемым спектральной кривой $Q(\lambda, \mu)=0$ пары коммутирующих операторов: $L \psi=\lambda \psi, M \psi=\mu \psi$, а общие собственные функции коммутирующих операторов задают $r$-мерное векторное расслоение над спектральной кривой (размерность $r$ векторного расслоения общих собственных функций пары коммутирующих операторов над спектральной кривой называется рангом коммутирующей пары операторов; ранг пары коммутирующих операторов является общим делителем порядков этих коммутирующих операторов). Первые примеры коммутирующих обыкновенных скалярных дифференциальных операторов нетривиальных рангов 2 и 3 и нетривиального рода $g=1$ были построены Диксмье [2] для неособой эллиптической спектральной кривой $\mu^{2}=\lambda^{3}-\alpha$, где $\alpha$ - произвольная ненулевая константа:

$$
\begin{gathered}
L=\left(\left(\frac{d}{d x}\right)^{2}+x^{3}+\alpha\right)^{2}+2 x \\
M=\left(\left(\frac{d}{d x}\right)^{2}+x^{3}+\alpha\right)^{3}+3 x\left(\frac{d}{d x}\right)^{2}+3 \frac{d}{d x}+3 x\left(x^{3}+\alpha\right)
\end{gathered}
$$

- коммутирующая пара операторов Диксмье ранга 2 рода $1, M^{2}=L^{3}-\alpha,[L, M]=0$;

$$
\begin{gathered}
L=\left(\left(\frac{d}{d x}\right)^{3}+x^{2}+\alpha\right)^{2}+2 \frac{d}{d x}, \\
M=\left(\left(\frac{d}{d x}\right)^{3}+x^{2}+\alpha\right)^{3}+3\left(\frac{d}{d x}\right)^{4}+3\left(x^{2}+\alpha\right) \frac{d}{d x}+3 x
\end{gathered}
$$

- коммутирующая пара операторов Диксмье ранга 3 рода $1, M^{2}=L^{3}-\alpha,[L, M]=0$. Эти замечательные примеры были получены Диксмье как коммутативные подалгебры алгебры

Работа выполнена при поддержке Российского фонда фундаментальных исследований (гранты №№ 11-01-00197-а, 11-01-12067-офи-м-2011) и программы "Ведущие научные школы" (грант № НШ-4995-2012.1).

DOI: $10.4213 /$ mzm9339 
Вейля $W_{1}$ [2]. Общая классификация коммутирующих обыкновенных скалярных дифференциальных операторов нетривиальных рангов $r>1$ была получена Кричевером [3]. Общий вид коммутирующих обыкновенных скалярных дифференциальных операторов ранга 2 для произвольной эллиптической спектральной кривой был найден Кричевером и Новиковым [4]. Выделение коммутирующих обыкновенных скалярных дифференциальных операторов с полиномиальными коэффициентами (коммутативных подалгебр алгебры Вейля $\left.W_{1}\right)$ является отдельной нетривиальной задачей, и полностью эта задача не решена даже для коммутирующих операторов Кричевера-Новикова ранга 2 рода 1, которые рационально параметризуются одной произвольной функцией (эта проблема была рассмотрена и изучена в [5], [6]). Мы также посвятим этому очень интересному вопросу отдельную статью. Функциональный параметр, отвечающий примеру Диксмье ранга 2 рода 1 среди всех коммутирующих операторов Кричевера-Новикова ранга 2 рода 1, найден Гриневичем [7]. Общий вид коммутирующих обыкновенных скалярных дифференциальных операторов ранга 3 для произвольной эллиптической спектральной кривой (общие коммутирующие операторы ранга 3 рода 1 параметризуются двумя произвольными функциями) был получен Моховым [8], [9], где также найдены функциональные параметры, отвечающие примеру Диксмье ранга 3 рода 1 среди всех коммутирующих операторов ранга 3 рода 1 . Кроме того, для любого ранга $r$ были построены примеры коммутирующих обыкновенных скалярных дифференциальных операторов рода 1 с полиномиальными коэффициентами (некоторые коммутативные подалгебры алгебры Вейля $W_{1}$ ) (см. также [10]). Отметим, что коэффициенты одного из пары коммутирующих операторов всегда выражаются полиномиально через коэффициенты второго оператора этой коммутирующей пары и их производные. Недавно Миронов [11] (см. также более ранние статьи [12]-[14]) построил для любого рода $g>1$ замечательные примеры коммутирующих обыкновенных скалярных дифференциальных операторов рангов 2 и 3 с полиномиальными коэффициентами, естественно обобщающие примеры Диксмье рангов 2 и 3 рода 1:

$$
\begin{aligned}
& L=\left(\left(\frac{d}{d x}\right)^{2}+x^{3}+\alpha\right)^{2}+g(g+1) x, \\
& M^{2}=L^{2 g+1}+a_{2 g} L^{2 g}+\cdots+a_{1} L+a_{0},
\end{aligned}
$$

где $a_{i}$ - некоторые константы, $\alpha$ - произвольная ненулевая константа, $L$ и $M$ - коммутирующие операторы Миронова ранга 2 рода $g$ (порядки операторов 4 и $4 g+2$ ), коэффициенты оператора $M$ выражаются полиномиально через коэффициенты оператора $L$ и их производные, $[L, M]=0$;

$$
\begin{aligned}
& L=\left(\left(\frac{d}{d x}\right)^{3}+x^{2}+\alpha\right)^{2}+g(g+1) \frac{d}{d x}, \\
& M^{2}=L^{2 g+1}+a_{2 g} L^{2 g}+\cdots+a_{1} L+a_{0},
\end{aligned}
$$

где $a_{i}$ - некоторые константы, $\alpha$ - произвольная ненулевая константа, $L$ и $M$ - коммутирующие операторы Миронова ранга 3 рода $g$ (порядки операторов 6 и $6 g+3$ ), коэффициенты оператора $M$ выражаются полиномиально через коэффициенты оператора $L$ и их производные, $[L, M]=0$.

Построим примеры коммутирующих обыкновенных скалярных дифференциальных операторов с полиномиальными коэффициентами, отвечающих спектральной кривой произвольного рода $g$ для произвольного четного ранга $r=2 k, k>1$.

Теорема 1. Операторы $L$ и $M$ порлдков $4 k u 4 k g+2 k$ соответственно,

$$
\begin{gathered}
L=\left(\left(\frac{d}{d x}\right)^{2 k}-2 x\left(\frac{d}{d x}\right)^{k}-k\left(\frac{d}{d x}\right)^{k-1}+\left(\frac{d}{d x}\right)^{3}+x^{2}+\alpha\right)^{2}+g(g+1) \frac{d}{d x}, \\
M^{2}=L^{2 g+1}+a_{2 g} L^{2 g}+\cdots+a_{1} L+a_{0},
\end{gathered}
$$


где $a_{i}$ - некоторые константы, $\alpha$ - произвольная ненулевая константа, являются коммутирующими операторами ранга $r=2 k, k>1$, рода $g,[L, M]=0$, коэффициенты оператора $M$ выражаются полиномиально через коэфбициенты оператора $L$ и их производные. При $k>2$ коммутирующие операторы $L$ и $M$ имеют стандартный (канонический) вид.

Построим примеры коммутирующих обыкновенных скалярных дифференциальных операторов с полиномиальными коэффициентами, отвечающих спектральной кривой произвольного рода $g$ для произвольного ранга вида $r=3 k, k \geqslant 1$.

Теорема 2. Операторы $L$ и $M$ порядков $6 k u 6 k g+3 k$ соответственно,

$$
\begin{gathered}
L=\left(\left(\frac{d}{d x}\right)^{3 k}-3 x\left(\frac{d}{d x}\right)^{2 k}-3 k\left(\frac{d}{d x}\right)^{2 k-1}+3 x^{2}\left(\frac{d}{d x}\right)^{k}+3 k x\left(\frac{d}{d x}\right)^{k-1}\right. \\
\left.+k(k-1)\left(\frac{d}{d x}\right)^{k-2}+\left(\frac{d}{d x}\right)^{2}-x^{3}+\alpha\right)^{2}-g(g+1) x \\
M^{2}=L^{2 g+1}+a_{2 g} L^{2 g}+\cdots+a_{1} L+a_{0},
\end{gathered}
$$

где $a_{i}$ - некоторые константы, $\alpha$ - произвольная ненулевая константа, являются коммутирующими операторами ранга $3 k, k \geqslant 1$, рода $g,[L, M]=0$, коэффициенты оператора $M$ выражаются полиномиально через коэффициенты оператора $L$ u их производные. При $k>1$ коммутирующие операторы $L$ и $M$ имеют стандартный (канонический) вид.

\section{СПИСОК ЦИТИРОВАННОЙ ЛИТЕРАТУРЫ}

[1] J. L. Burchnall, I. W. Chaundy, Proc. London Math. Soc. (2), 21 (1923), 420-440. [2] J. Dixmier, Bull. Soc. Math. France, 96 (1968), 209-242. [3] И. М. Кричевер, Функи. анализ и его прил., 12:3 (1978), 20-31. [4] И. М. Кричевер, С.П. Новиков, ДАН СССР, 247:1 (1979), 33-37. [5] O. I. Mokhov, International Conference on Algebra in Memory of A. I. Shirshov (1921-1981), Reports on Theory of Rings, Algebras and Modules (Barnaul, USSR, 20-25 August 1991), 1991, 85. [6] O.I. Mokhov, Third International Conference on Algebra in Memory of M.I. Kargapolov (1928-1976) (Krasnoyarsk, Russia, 23-28 August 1993), Inoprof, Krasnoyarsk, 1993, 421. [7] П. Г. Гриневич, Функи. анализ и его прил., 16:1 (1982), 19-24. [8] О.И. Мохов, УМН, 37:4(226) (1982), 169-170. [9] О. И. Мохов, Изв. АН СССР. Cер. матем., 53:6 (1989), 1291-1315. [10] P. Dehornoy, Compositio Math., 43:1 (1981), 71-99. [11] A.E. Mironov, Self-Adjoint Commuting Differential Operators and Commutative Subalgebras of the Weyl Algebra, arXiv: 1107.3356. [12] А. Е. Миронов, Сиб. электрон. матем. изв., 6 (2009), 533-536. [13] А.Е. Миронов, Функи. анализ и его прил., 39:3 (2005), 91-94. [14] А.Е. Миронов, Матем. сб., 195:5 (2004), 103-114.

О. И. Мохов

Поступило

Московский государственный университет

31.01 .2012

им. М. В. Ломоносова

E-mail: mokhov@mi.ras.ru,

mokhov@landau.ac.ru, mokhov@bk.ru 